\title{
Photocatalytic Oxidation of a Volatile Organic Component of Acetaldehyde Using Titanium Oxide Nanotubes
}

\author{
Huifang Xu, ${ }^{1}$ Ganesh Vanamu, ${ }^{2}$ Ziming Nie, ${ }^{1}$ Hiromi Konishi, ${ }^{1}$ Rakesh Yeredla, ${ }^{1}$ \\ Jonathan Phillips, ${ }^{3}$ and Yifeng Wang ${ }^{4}$ \\ ${ }^{1}$ Department of Geology and Geophysics and Material Science Program, University of Wisconsin, 1215 West Dayton Street, \\ Madison, WI 53706, USA \\ ${ }^{2}$ Department of Chemical and Nuclear Engineering, University of New Mexico, Albuquerque, NM 87131, USA \\ ${ }^{3}$ Los Alamos National Laboratory, Engineering Science and Applications Division, MS C390, Los Alamos, NM 87545, USA \\ ${ }^{4}$ Sandia National Laboratories, P.O. Box 5800, Albuquerque, NM 87185, USA
}

Received 14 February 2006; Revised 9 November 2006; Accepted 22 December 2006

Titanium oxide nanotubes are prepared and treated with $\mathrm{Au}(\mathrm{Au} /$ nanotube sample) and $\mathrm{Pt}(\mathrm{Pt} /$ nanotube sample), and the photoactivity of these catalysts compared to a standard Degussa P25 photocatalyst is investigated. The samples were analyzed using $\mathrm{X}$-ray diffraction, field emission gun scanning transmission electron microscopy (STEM). Both high-resolution TEM images and high-angle annular dark-field (HAAD) images were recorded for the specimens. Oxidation of acetaldehyde was used to test the efficiency of the catalysts. Nanotube samples showed better photoactivity than the standard P25, because the P25 titania deactivates quickly. Enhanced reactivity of the nanotube is related to surface charge polarity developed on outer and inner surfaces due to the difference in overlap of oxygen anions that resulted from curving of octahedral sheets. A tentative and qualitative surface polarity model is proposed for enhancing electron-hole pair separation. The inner surface benefits reduction; whereas, the outer surface benefits oxidation reactions. Both the metal identity and the size of the metal particles in the nanotubes affected the photocatalytic activity. Specifically, the addition of platinum increased the activity significantly, and increased the total yield. The addition of gold had lesser impact compared to the platinum. Formation of Pt large nanoparticles on the nanotube surfaces reduces the oxidation reactivity.

Copyright (c) 2006 Huifang Xu et al. This is an open access article distributed under the Creative Commons Attribution License, which permits unrestricted use, distribution, and reproduction in any medium, provided the original work is properly cited.

\section{INTRODUCTION}

Interest in photocatalysis continues to grow [1-4], reflecting the demonstrated success of these catalysts for decontamination and purification of water and air polluted by volatile organic compounds [5-7]. In particular, illuminated semiconductors have been used to oxidize compounds typically found in low concentrations in industrial effluent streams including alkanes, alkenes, phenols, aromatic acids, and surfactants [8-13]. Heterogeneous photocatalytic systems have also been used to recover metals, for example, from reductive deposition of heavy metals from wastewater streams, and to produce novel well-dispersed metal-loaded semiconductor catalysts by photodeposition of metallic ions [14].

Among photocatalytic materials, titanium oxide is believed to be the most promising, due to its great capacity for oxidation, wide band gap, nontoxicity, low cost, widespread availability, and long term stability [15]. Photocatalytic reactions involving titanium dioxide have been studied since the 1920s when Kiedel studied the roles of titania (called "titanium white pigment" for fading in paints [16]. Interest in titania photocatalysis increased dramatically after Fujishima and Honda [1], in 1972, discovered that a rutile titanium dioxide electrode could split water illuminated by near UV light.

For the present work, we tested the photocatalytic efficiency of a new form of titanium oxide, metal-doped and metal-coated Ti-oxide nanotubes, for the oxidation of acetaldehyde [17-19] and demonstrated that these novel catalysts are better than the industrial standard P25 in longterm run. Also, following the positive results of recent literature [20-22], we tested the impact of metal addition by standard wet impregnation and found that platinum increased the maximum activity significantly, whereas gold had lesser impact. Earlier studies that showed a benefit with metal addition required the use of ion implantation or plasma processes. The present process for metal addition is clearly simpler and less costly. 


\section{SAMPLES AND EXPERIMENTAL METHODS}

To test the impact of various treatments on the photoreactive performance of titanium oxides, we studied batches of four samples: (1) P25 (Degussa), (2) Titanium oxide nanotube, (3) Au nanoparticle-coated nanotube (Au/nanotube), and (4) Pt-treated nanotube (Pt/nanotube). Titanium oxide nanotubes are prepared by adding $1 \mathrm{~g}$ of $\mathrm{P} 25$ powder to $60 \mathrm{ml} 10 \mathrm{M} \mathrm{NaOH}$ solution and annealing at $150^{\circ} \mathrm{C}$ in digestion bomb for 20 hours [23]. The products were washed using distilled water until water became neutral. Au/nanotube samples were prepared by mixing $0.5 \mathrm{~g}$ of wet nanotube sample and $20 \mathrm{ml} \mathrm{HAuCl}_{4}$ solution (10 wt\% Au), stirring for 30 minutes at ambient temperature. The mixture was then centrifuged to separate liquid and powder components. The powder product was washed using distilled water 5 times, dried at $110^{\circ} \mathrm{C}$ for 10 hours and finally, ground into fine powder. $\mathrm{Pt} /$ nanotube samples were prepared in a similar fashion only substituting $\mathrm{H}_{2} \mathrm{PtCl}_{6}$ solution (10 wt $\% \mathrm{Pt}$ ), for the gold compound.

The specific surface area and pore size characterization are done using a NOVA 2200 surface area and pore size analyzer (quantachrome instruments) using nitrogen gas as the adsorbate. The samples were degassed at $230^{\circ} \mathrm{C}$ for 20 hours before analysis. X-ray powder diffraction analyses were carried out by using a powder X-ray diffraction unit (Scintag Pad V with a Ge solid-state detector; $\mathrm{Cu} \mathrm{K}_{\alpha}$ radiation) with the solid specimens mounted on a low background quartz holder.

Both TEM and STEM analyses were carried out by using a Tecnai F30 field emission gun scanning transmission electron microscope equipped with an X-ray energydispersive spectroscopy (EDS) system (EM Vision 4.0), and a JEOL FEG-2010F field emission gun scanning transmission electron microscope (STEM) with attached Oxford instruments' X-ray energy-dispersive spectroscopy (EDS) system and Gatan imaging filtering (GIF) system. Both highresolution TEM images and high-angle annular dark-field (HAAD) images were recorded for the specimens. HAAD imaging is a chemical sensitive technique. The local intensity of a HAAD image is approximately proportional to the square of local average atomic number $(Z)$ [24, 25]. Therefore, it is very sensitive to detect "bright" clusters and nanoparticles of heavy metals (like Au and $\mathrm{Pt}$ ).

The photocatalytic oxidation of acetaldehyde was done using a continuous flow reactor [26] with same flow rates for all the experiments. As shown in Figure 1, helium is passed through a sparger that was kept at 0 degree using ice and water mixture in order to carry the saturated acetaldehyde to the annular photoreactor. Oxygen was set to $20 \%$ of the total flow for all the experiments. In all cases, $25 \mathrm{mg}$ of catalyst was evenly dispersed on the inner surface of the outer tube of the $12 \mathrm{~cm}$ long, $3 \mathrm{~cm}$ outer diameter, annular reactor. We placed suspensions of the photocatalysts and acetone on inner surfaces of the reactor tubes and rolled the tubes until the suspensions dry. The reactor (see Figure 2) has a total volume of nearly $13.3 \mathrm{~cm}^{3}$. The inner tube is the source of the light, a TL 8 W "black light" (Philips, Made in Holland). The space

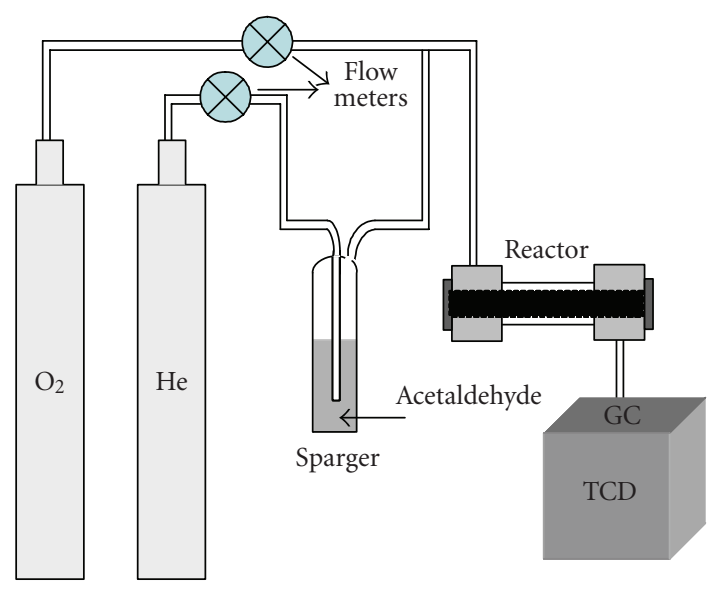

FIGURE 1: Experimental setup for the continuous photocatalysis of oxidation of acetaldehyde.

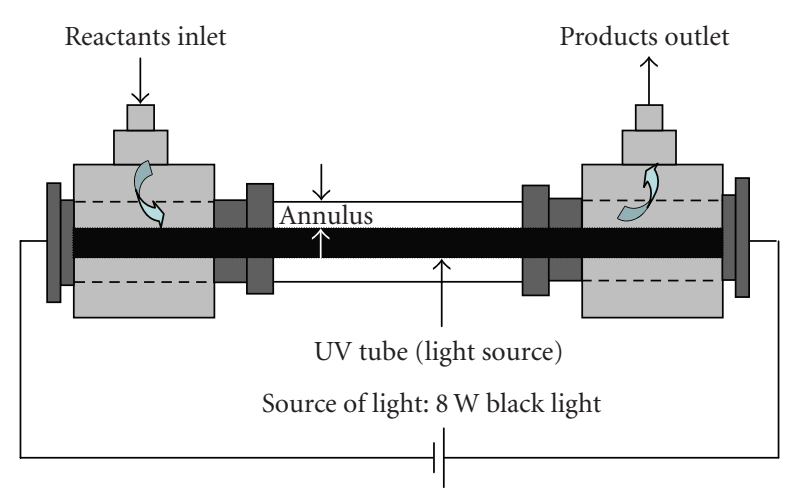

FIgURE 2: Schematic of the annular reactor.

from the lamp to the catalyst film is approximately $1 \mathrm{~mm}$, which will ensure that gas phase mass transfer effects are minimized. Temperature measurement with a thermocouple showed that during operation the catalyst was at about $50^{\circ} \mathrm{C}$.

Both product and reactant (sequentially in all cases) analysis was performed using the thermal conductivity detector function of an HP 5890 series II gas chromatography (GC) equipped with a 10 -port valve. A Haysep D column (8 feet $\times 1 / 8$ inch SS) was employed to separate acetaldehyde, acetic acid, carbon dioxide, carbon monoxide, and water at ambient temperature. All the experiments were carried at similar conditions. The purpose of the experiments is to obtain relative reactivity of the nanotube-based photocatalysts with respect to $\mathrm{P} 25$ titania.

\section{RESULTS}

Figure 3 shows the TEM image of the pure titanium oxide nanotubes (top) and a reference photocatalyst of Degussa P25 titania (bottom). It can be seen that the nanotube sample consists of low-defect density nanotubes virtually all of which have an inner diameter of $\sim 4-6 \mathrm{~nm}$. This nanotube is basically Na-titanate nanotube with chemical formula of 


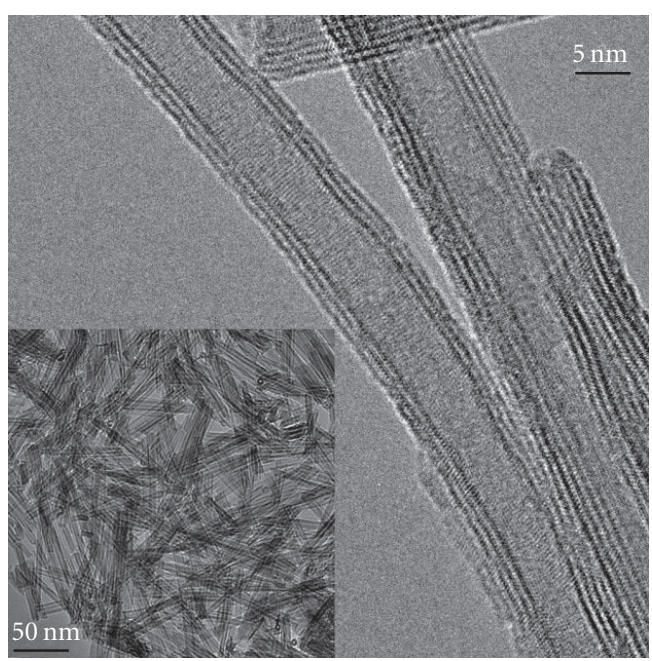

(a)

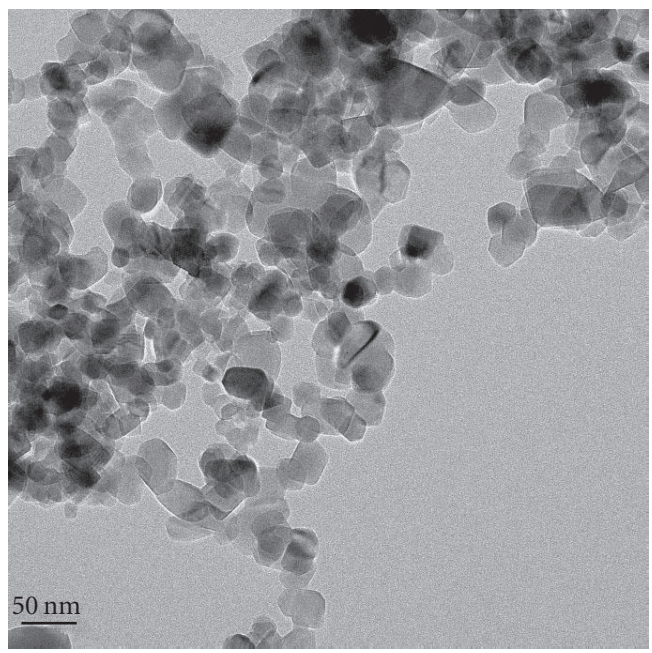

(b)

FIGURE 3: (a) High-resolution TEM image and bright-field TEM image (insert at the low-left corner) of the pure titanium oxide nanotube; (b) bright-field TEM image of the P25 titania with size range of about 20-60 nm.

$(\mathrm{Na}, \mathrm{H})_{2} \mathrm{Ti}_{3} \mathrm{O}_{7}[27,28]$. P25 titania is a mixture of anatase $(84 \%)$ and rutile (16\%) nanocrystals based on our quantitative X-ray analysis using Rietveld method with a software GSAS (see Figure 4). X-ray diffraction pattern from the nanotube sample displays very broad peaks due to curved (001) lattice planes and thin tube walls (see Figure 5). The measured surface areas using multipoint BET method for the P25 titania and nanotube samples are $61 \mathrm{~m}^{2} / \mathrm{g}$, and $149 \mathrm{~m}^{2} / \mathrm{g}$, respectively.

Figure 6(a) shows the HAAD image of Au/nanotube sample. It clearly shows Au nanoparticles (bright spots) with diameter of $\sim 1 \mathrm{~nm}$ attached to the nanotube surfaces. Figure 6(b) shows the HAAD image of Pt/nanotube sample. The HAAD image does not show visible Pt clusters on the nanotube surfaces. However, X-ray EDS spectra clearly show

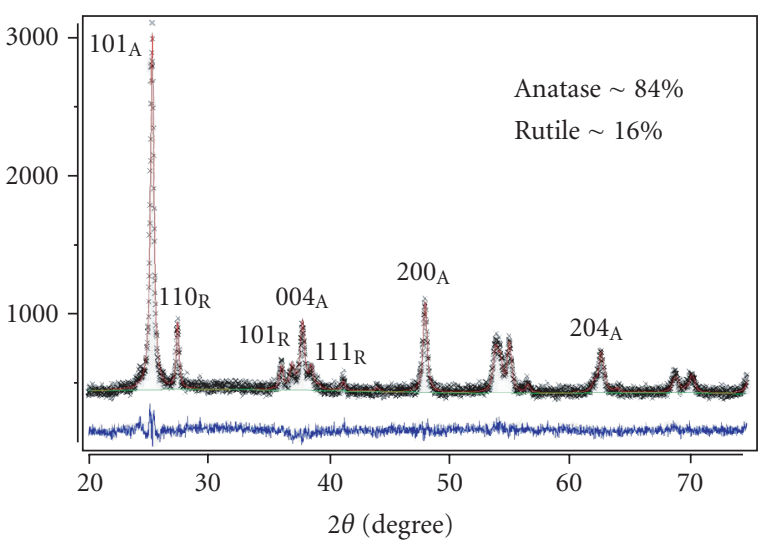

Figure 4: X-ray powder diffraction pattern from the P25 titania photocatalyst. Quantitative analysis using Rietveld method shows that photocatalyst contains $84 \%$ of anatase and $16 \%$ of rutile.

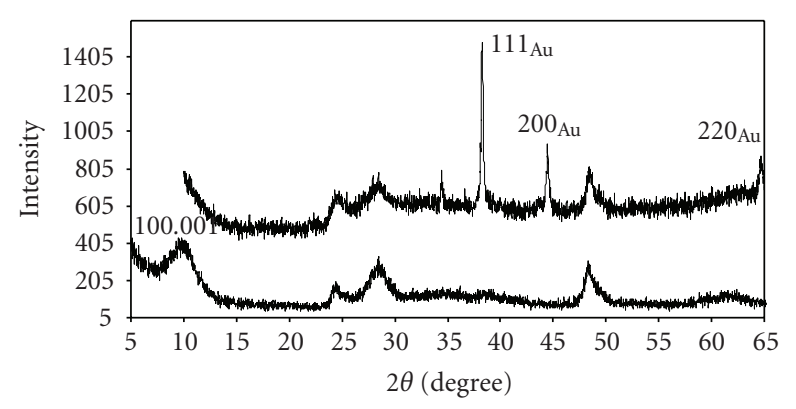

Figure 5: X-ray powder diffraction pattern of the Ti-oxide nanotube (bottom), and Au-treated nanotube sample with large gold nanocrystals (upper) showing broad diffraction peaks due to curving of (001) lattice planes and thin crystal sheets from the nanotubes and relatively sharp peaks from gold.

the $\mathrm{Pt}$ in the nanotubes. It is suggested that $\mathrm{Pt}$ (that may be sorbed on nanotube surface in forms of cation and/or metal complex) are distributed evenly on both outer and inner surfaces of the nanotubes. In order to test the effect of nanoparticle size on the photo reactivity, we prepared the nanotubes coated with large $\mathrm{Au}$ and $\mathrm{Pt}$ particles by heating the $\mathrm{Au}$ and Pt treated nanotubes at $150^{\circ} \mathrm{C}$ for over night). HAAD images show relatively large Au and Pt nanocrystals (see Figure 7). An XRD pattern from the Au-coated nanotube sample clearly displays strong peaks from gold nanocrystals (see Figure 5).

Reactivity testing showed that all the novel catalysts studied are better (per gram) than the standard titania catalysts, P25 from Degussa, for the oxidation reaction studied. Acetaldehyde was readily oxidized to carbon dioxide at room temperature on all titania samples when the UV lights were turned on, as shown in Figure 8. In all the cases, the rate of photocatalytic oxidation (per gram) was plotted as a function of time. Except for the pure nanotube sample, all other photocatalysts decrease in their reactivity. Indeed, deactivation during hydrocarbon oxidation processes has been noted and studied repeatedly for high concentration experiments in 


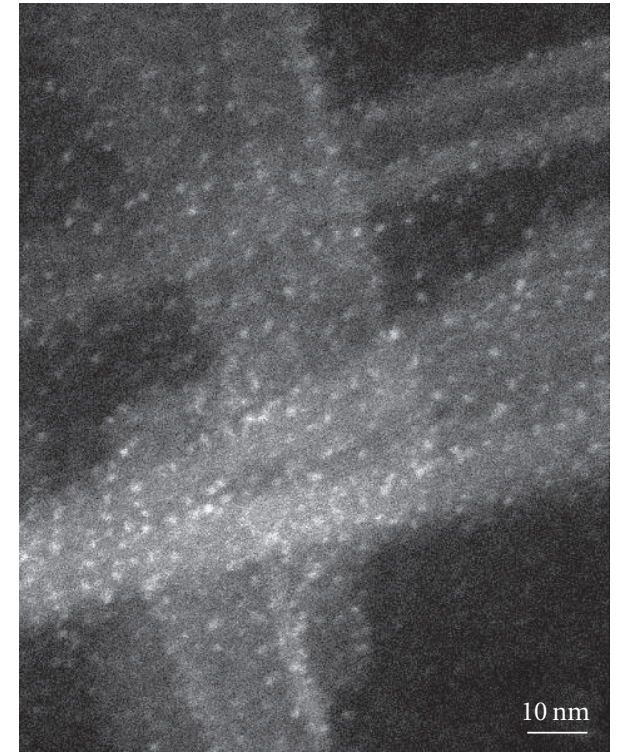

(a)

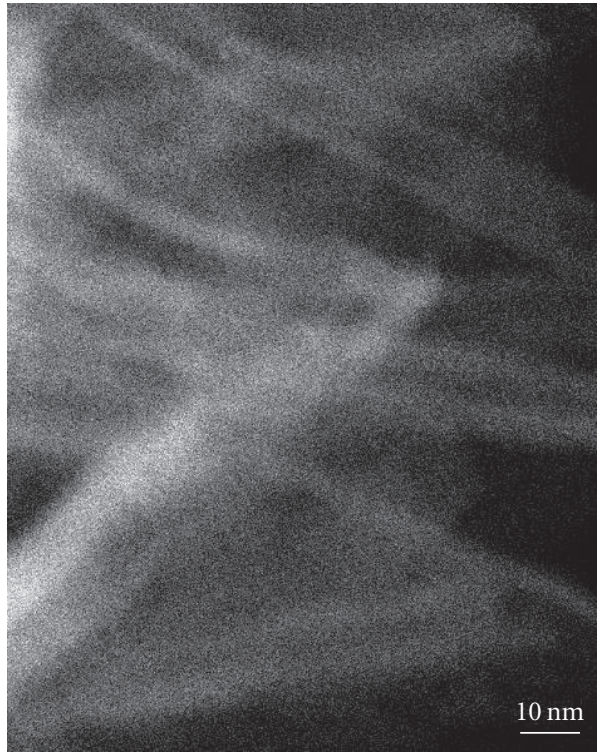

(b)

FIGURE 6: HAAD images of the Au-treated nanotube sample (a) and Pt-treated nanotube sample (b).

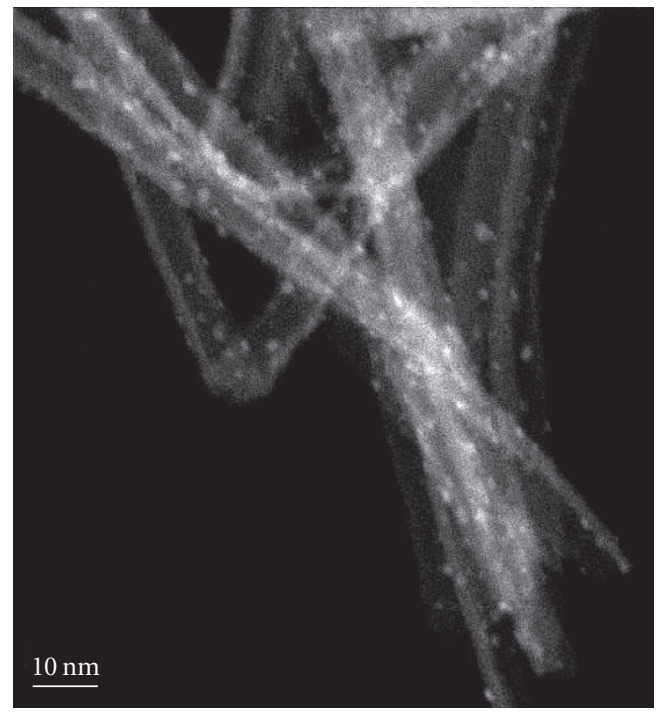

(a)

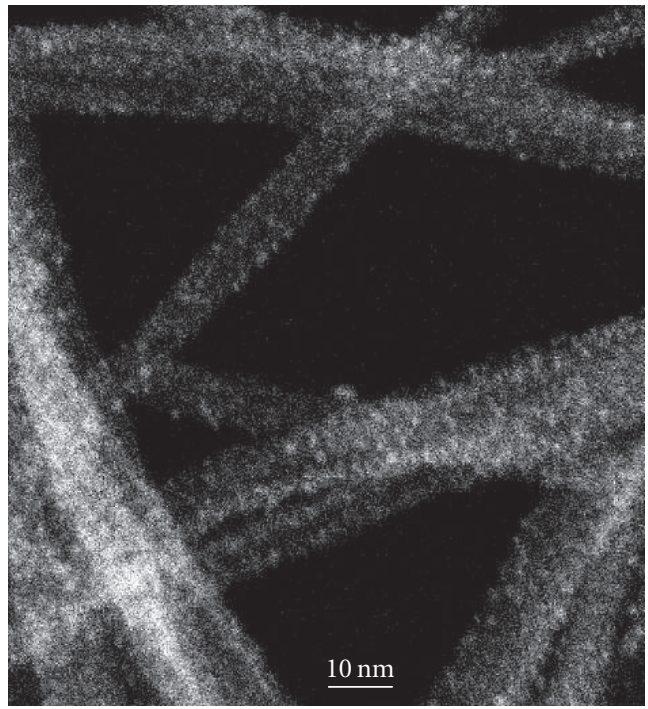

(b)

FIGURE 7: HAAD scanning transmission electron microscopic (STEM) images of the nanotube samples with large Au particles (a) and the sample with large Pt particles (b).

recent years $[29,30]$ and there are several proposals for the mechanism of this deactivation [31].

As noted above, all the catalysts were more active than the standard material, P25. In the case of nanotube without metal addition, the activity is similar based on same weight and less reactive based on per unit surface area, initially. However, P25 rapidly deactivates and the nanotube photocatalytic activity actually increases slightly. Pt containing nanotube is a dramatically better catalyst than P25. The highest activity of this catalyst is more than 10 times the maximum rate of $\mathrm{P} 25$, and more than 6 times maximum rate of the pure nanotube sample. It must be noted that the reported relative rates closely reflect relative intrinsic rates with respect to P25 titania. After the reaction, the white $\mathrm{Pt} /$ nanotube specimen became grayish. Our TEM results show some Pt nanodots $(<1 \mathrm{~nm})$ formed on the nanotube surfaces (see Figure 9). It is suggested that the nanotubes with uniformly distributed Pt (ions and metal complex) are 


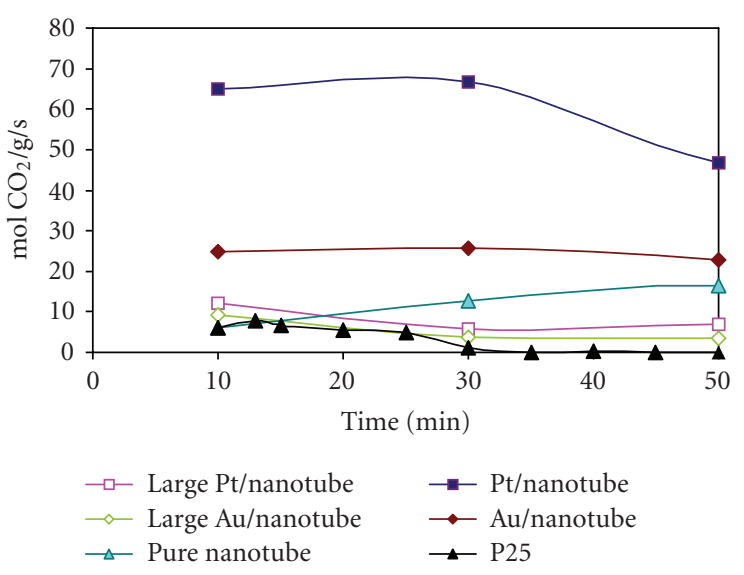

FIGURE 8: Photocatalytic oxidation rates of various titanium-oxidebased catalysts.

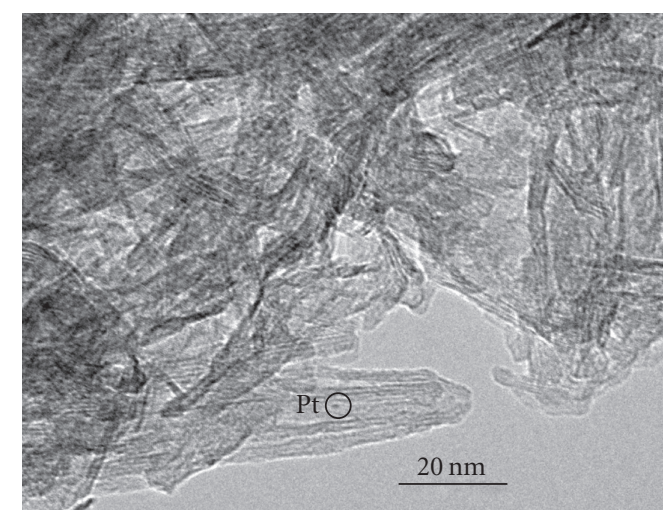

FIgURE 9: TEM image showing Pt dots without crystalline feature on the nanotube after photocatalytic reaction of the $\mathrm{Pt} /$ nanotube sample. The nanotubes become less regular in shape after treatment in acidic solutions.

more photo reactive than those with large Pt nanoparticles. The maximum activity of Au containing nanotube was not as high as that of the platinum-bearing sample, but still it reached an activity more than twice the maximum rate of the pure nanotube sample. It is also found that the reactivity decreased with larger particles. The nanotube samples coated with about $2 \sim 4 \mathrm{~nm}$ Au and Pt nanoparticles (see Figure 7) showed lower photo-reactive rates compared to the $\mathrm{Au} / \mathrm{nanotube}$ and $\mathrm{Pt} /$ nanotube samples (see Figure 8).

\section{DISCUSSIONS}

Initial activity studies of the photocatalytic activity of titanium oxide nanotubes showed that these materials have the potential for widespread application. Indeed, the measured activity for "neat" nanotubes for photocatalytic oxidation (PCO) of low concentrations of acetaldehyde at room temperature showed them to be more active than the standard material, P25. Metal addition, by a standard wet impregnation process, particularly platinum addition, increased the activity to the point that it is more than an order of magnitude better than the standard. Many more studies are required to understand the characteristic of these materials that increases the activity so dramatically.

It is generally believed that the photocatalytic process occurs because light of sufficient energy (e.g., greater than the optical band gap of Ti-oxides, 3-3.2 eV, i.e., 388-413 nm) excites electrons in semiconductors from the valence band to the conduction band that can either recombine and release great amounts of energy, or migrate to the surface where they are available for reduction and oxidation reactions by charge transfer to species adsorbed onto the semiconductor surface.

The mechanism of $\mathrm{TiO}_{2}$ photocatalyzed reactions is that superoxide, $\mathrm{O}_{2}{ }^{-}$, and specifically, hydroxyl radicals, ${ }^{\bullet} \mathrm{OH}$, act as active reagents for the mineralization of the organic compounds $[15,17]$. The radicals are formed by the scavenging of the electron-hole pair by molecular oxygen and water following equation (1):

$$
\begin{gathered}
\mathrm{O}_{2}+\mathrm{e}_{\mathrm{cb}}^{-} \longrightarrow \mathrm{O}_{2}^{\cdot-}, \\
\mathrm{OH}^{-}+\mathrm{h}^{+} \longrightarrow{ }^{\cdot} \mathrm{OH} .
\end{gathered}
$$

Insertion of $\cdot \mathrm{OH}$ radicals into $\mathrm{C}-\mathrm{H}$ bonds leads ultimately to the complete oxidation of the organic substrate. Indeed, complete mineralization of a variety of organic compounds, such as phenols, hydrocarbons, carboxylic acids, and chlorinated aliphatic and olefinic compounds, has been realized. Thus, as mentioned above, one feature of photocatalysts that impacts activity is the structure of the absorption band edge and separation of the electron-hole pairs. Small modifications in the impurity concentration or structure of titania are known to effect the structure of the absorption band edge. As the nanotubes are neither rutile nor anatase $[27,28]$, the two phases of pure titania and the structures are generally believed to be the best photocatalysts, the adsorption band edge of the nanotubes could be different, and that could account for its high observed reactivity. Our recent study shows that the band gap of the nanotube $(3.1 \mathrm{eV})$ is very close to that of anatase $(3.2 \mathrm{eV})$. It is also proposed that the surface of curved octahedral sheets of the nanotubes may be more reactive than normal titania crystal surface due to electrical polarity developed between the inner and outer surfaces.

The deprotonation/prototation reaction on oxide surface in aqueous solution can be simplified as

$$
>\mathrm{SOH}^{+0.5}=>\mathrm{SO}^{-0.5}+\mathrm{H}^{+},
$$

where $>\mathrm{SOH}^{+0.5}$ is protonated (positively charged) surface site, and $>\mathrm{SO}^{-0.5}$ is deprotonated (negatively charged) surface site [32-35]. Its equilibrium constant $K$ will be expressed as

$$
\mathrm{K}=\left[\mathrm{SO}^{-0.5}\right]\left[\mathrm{H}^{+}\right] /\left[>\mathrm{SOH}^{+0.5}\right],
$$

where $K$ can be related to $p K$ by a relationship of

$$
p K=-\log K \text {. }
$$




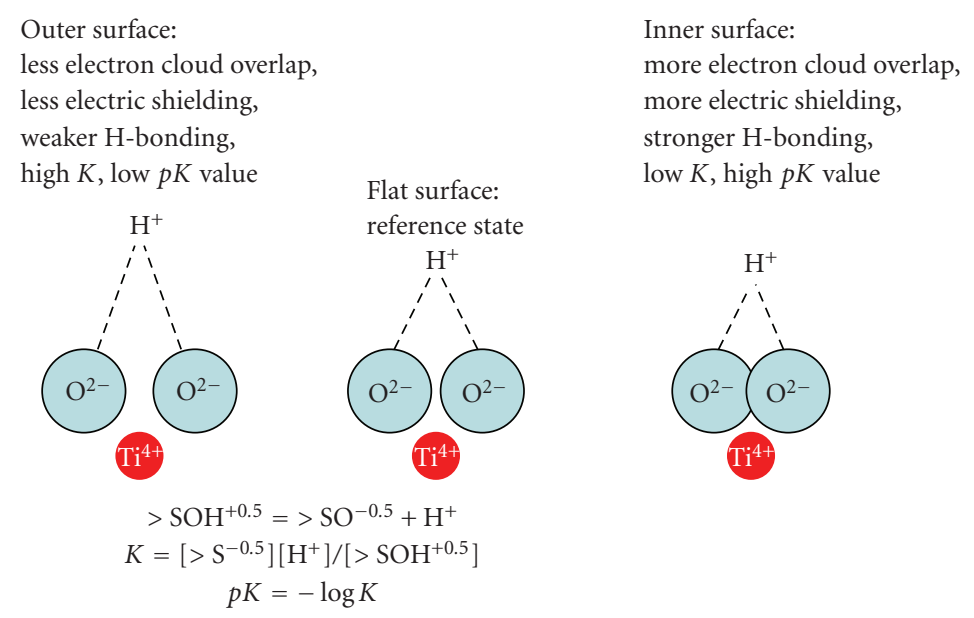

FIGURE 10: Models illustrate oxygen ions on inner surface (right) and outer surface (left) of the nanotube. The degree of electron clouds overlap between neighboring oxygen directly affect electric charge shielding from $\mathrm{Ti}^{4+}$, and therefore determines the bonding strength between $\mathrm{H}+$ and surface oxygen. "> S" represents surface of the nanotube.

When the octahedral sheets (or nanoplates) are rolled into nanotubes, the inner surface oxygen atoms will be closer than those on flat surface, which will result in more electron clouds overlaps, and shielding from $\mathrm{Ti}^{4+}$ in the center of octahedra. This will result in strong bonding between $\mathrm{H}^{+}$ and surface oxygen, or more protonated surface sites (see Figure 10). The effect will be opposite on the outer surface (with positive curvature). This will result in difference in surface protonation or surface acidity, that is, $p K$ value of the inner surface will be higher than that of flat surface; and the $p K$ value of outer surface will be lower than that of flat surfaces. Based on the proposed mechanism in Figure 10, it can be inferred that the inner surface of the nanotube has high acidity, and the outer surface has lower acidity.

The different surface acidities will result in different charges on inner and outer surfaces, or electrical polarity. In a solution at certain $\mathrm{pH}$ conditions, outer surface will display net negative charge and inner surface will display net positive charge (see Figure 11). The electrical polarity will enhance electron-hole separation, and therefore leading positive holes in the valence band to the outer surface for photo-catalytic oxidation, and electrons in the conduction band to the inner surface for photo-catalytic reduction and even reduction of water to hydrogen (see Figure 11).

Our data from photo-catalytic oxidation of acetaldehyde (a VOC) shows that reactivity of the nanotube and Degussa P25 titania is very similar at the beginning (relatively dry) based on same weight (see Figure 8). After 30 minutes of reaction, the reactivity of P25 decreases dramatically. However, the reactivity of nanotube increases (see Figure 8 ). We propose that the development of electrical polarity on the nanotube surface in aqueous system enhances the charge separation and photocatalytic oxidation (see Figures 10 and 11). The oxidation of acetaldehyde will produce water and carbon dioxide through the net reaction of

$$
\mathrm{CH}_{3} \mathrm{CHO}+2 \mathrm{O}_{2}=2 \mathrm{CO}_{2}+2 \mathrm{H}_{2} \mathrm{O} \text {. }
$$



FIgure 11: A diagram showing Ti-oxide nanotube with positive charges on inner surface and negative charges on outer surface in aqueous solution, which will enhance charge separation of electronhole pairs (i.e., migration of negatively charged electrons to the inner surface and positively charged holes to the outer surface). See reactions (1) for generation of radicals by incorporating electrons and holes, $\mathrm{CB}=$ conduction band, $\mathrm{VB}=$ valence band.

Water and $\mathrm{CO}_{2}$ will result in weak acidic solution sorbed on the nanotube surfaces, which enhances surface polarity and drives the oxidation further.

The testing of the impact of metal additives in the present work reflects recent findings by others. For example, Anpo et al. [17] showed that metal ion implantation can dramatically change the photo-activity of titania for NO reduction. Other works $[20,21]$ indicate that "plasma treated" doped titania shows improved performance for some photoactivated processes. Yet, more recent works [21, 22] suggest 
that N-doped titania is a more effective catalyst for some frequency ranges than undoped titania. Notably, all earlier studies used high-energy processes to add metal "dopants" to the lattice in order to modify the photoadsorption edge, per the theory discussed above. In several of these earlier studies, it was found that the high energy doping processes modified other characteristics of the material, notably the phase. Thus, simple correlations of changed activity with band-gap and surface reactivity are suspect. The results of the present paper show that simple, standard methods of metal addition (such as the $\mathrm{Pt} /$ nantotube sample), without the need for "ion implantation" or other complex and expensive processes, can dramatically improve the performance of titanium oxide for some (i.e., hydrocarbon oxidation) photocatalytic reactions, although the influence of "band edge" changes on photocatalytic activity is not clear.

\section{CONCLUSIONS}

The titanium oxide nanotube is a potential catalyst for photocatalytic oxidation of low concentrations of acetaldehyde at room temperature. The commercial P25 deactivates quickly in the photo-oxidation of acetaldehyde. The pure nanotube is more reactive than P25 titania in long run. The bending and curving of the $\mathrm{Ti}-\mathrm{O}$ octahedral sheets results in electrical polarity being developed on outer and inner surfaces, which enhances the electron-hole separation and the observed photo oxidation by the nanotubes. Both $\mathrm{Au}$ - and Pt-treated nanotube samples increase the photo reactivity. However, size of the $\mathrm{Au}$ and $\mathrm{Pt}$ nanoparticles on the nanotube surfaces likely affected the photo reactivity. Large size of the Au and Pt particles will decrease the photo reactivity. Specifically, the addition of platinum without formation of obvious nanoparticles on the nanotube surfaces increased the maximum activity significantly, and increased the total yield. The thin films of the titanium oxide nanotube could be further tested in a scaled-up photocatalytic reactor.

\section{ACKNOWLEDGMENTS}

This work was supported by NSF (EAR02-10820), the University of New Mexico, and the Graduate School of the University of Wisconsin-Madison. Authors thank three reviewers for critical comments and suggestions.

\section{REFERENCES}

[1] O. Legrini, E. Oliveros, and A. M. Braun, "Photochemical processes for water treatment," Chemical Reviews, vol. 93, no. 2, pp. 671-698, 1993.

[2] M. A. Fox and M. T. Dulay, "Heterogeneous photocatalysis," Chemical Reviews, vol. 93, no. 1, pp. 341-357, 1993.

[3] A. Hagfeldt and M. Graetzel, "Light-induced redox reactions in nanocrystalline systems," Chemical Reviews, vol. 95, no. 1, pp. 49-68, 1995.

[4] P. V. Kamat, "Photochemistry on nonreactive and reactive (semiconductor) surfaces," Chemical Reviews, vol. 93, no. 1, pp. 267-300, 1993.

[5] H. Gerischer and A. Heller, "Photocatalytic oxidation of organic molecules at $\mathrm{TiO}_{2}$ particles by sunlight in aerated water,"
Journal of the Electrochemical Society, vol. 139, no. 1, pp. 113118, 1992.

[6] N. B. Jackson, C. M. Wang, Z. Luo, et al., "Attachment of $\mathrm{TiO}_{2}$ powders to hollow glass microbeads. Activity of the $\mathrm{TiO}_{2}$ coated beads in the photoassisted oxidation of ethanol to acetaldehyde," Journal of the Electrochemical Society, vol. 138, no. 12, pp. 3660-3664, 1991.

[7] M. Nair, Z. Luo, and A. Heller, "Rates of photocatalytic oxidation of crude oil on salt water on buoyant, cenosphereattached titanium dioxide," Industrial and Engineering Chemistry Research, vol. 32, no. 10, pp. 2318-2323, 1993.

[8] R. Cai, K. Hashimoto, K. Itoh, Y. Kubota, and A. Fujishima, "Photokilling of malignant cells with ultrafine $\mathrm{TiO}_{2}$ powder," Bulletin of the Chemical Society of Japan, vol. 64, no. 4, pp. 1268-1273, 1991.

[9] G. Mills and M. R. Hoffmann, "Photocatalytic degradation of pentachlorophenol on titanium dioxide particles: identification of intermediates and mechanism of reaction," Environmental Science and Technology, vol. 27, no. 8, pp. 1681-1689, 1993.

[10] C. Kormann, D. W. Bahnemann, and M. R. Hoffmann, "Photolysis of chloroform and other organic molecules in aqueous titanium dioxide suspensions," Environmental Science and Technology, vol. 25, no. 3, pp. 494-500, 1991.

[11] A. Chemseddine and H. P. Boehm, "A study of the primary step in the photochemical degradation of acetic acid and chloroacetic acids on $\mathrm{TiO}_{2}$ photocatalyst," Journal of Molecular Catalysis, vol. 60, no. 3, pp. 295-311, 1990.

[12] H. Hidaka, J. Zhao, E. Pelizzetti, and N. Serpone, "Photodegradation of surfactants. 8. Comparison of photocatalytic processes between anionic DBS and cationic BDDAC on the titania surface," Journal of Physical Chemistry, vol. 96, no. 5, pp. 2226-2230, 1992.

[13] E. Pelizzetti, C. Minero, P. Piccinini, and M. Vincenti, "Phototransformations of nitrogen containing organic compounds over irradiated semiconductor metal oxides. Nitrobenzene and atrazine over $\mathrm{TiO}_{2}$ and $\mathrm{ZnO}$," Coordination Chemistry Reviews, vol. 125, no. 1-2, pp. 183-193, 1993.

[14] M. R. Prairie, L. R. Evans, B. M. Stange, and S. L. Martinez, "An investigation of titanium dioxide photocatalysis for the treatment of water contaminated with metals and organic chemicals," Environmental Science and Technology, vol. 27, no. 9, pp. 1776-1782, 1993.

[15] A. J. Bard, "Photoelectrochemistry," Science, vol. 207, no. 4427, pp. 139-144, 1980.

[16] E. Keidel, "Die Beeinflussung der Lichtechtheit von Teerfarblacken durch Titanweiss [Influence of titanium white on the fastness to light of coal-tar days]," Farben-Zeitung, vol. 34, pp. 1242-1243, 1929.

[17] M. Anpo, "Use of visible light. Second-generation titanium oxide photocatalysts prepared by the application of an advanced metal ion-implantation method," Pure and Applied Chemistry, vol. 72, no. 9, pp. 1787-1792, 2000.

[18] S. Luo and J. L. Falconer, "Aldol condensation of acetaldehyde to form high molecular weight compounds on $\mathrm{TiO}_{2}$," Catalysis Letters, vol. 57, no. 3, pp. 89-93, 1999.

[19] S. Luo and J. L. Falconer, "Acetone and acetaldehyde oligomerization on $\mathrm{TiO}_{2}$ surfaces," Journal of Catalysis, vol. 185, no. 2, pp. 393-407, 1999.

[20] H. Yamashita, M. Harada, J. Misaka, M. Takeuchi, K. Ikeue, and M. Anpo, "Degradation of propanol diluted in water under visible light irradiation using metal ion-implanted 
titanium dioxide photocatalysts," Journal of Photochemistry and Photobiology A: Chemistry, vol. 148, no. 1-3, pp. 257-261, 2002.

[21] I. Nakamura, N. Negishi, S. Kutsuna, T. Ihara, S. Sugihara, and K. Takeuchi, "Role of oxygen vacancy in the plasma-treated $\mathrm{TiO}_{2}$ photocatalyst with visible light activity for NO removal," Journal of Molecular Catalysis A: Chemical, vol. 161, no. 1-2, pp. 205-212, 2000.

[22] K. Takeuchi, I. Nakamura, O. Matsumoto, S. Sugihara, M. Ando, and T. Ihara, "Preparation of visible-light-responsive titanium oxide photocatalysts by plasma treatment," Chemistry Letters, vol. 29, no. 12, pp. 1354-1355, 2000.

[23] T. Kasuga, M. Hiramatsu, A. Hoson, T. Sekino, and K. Niihara, "Formation of titanium oxide nanotube," Langmuir, vol. 14, no. 12, pp. 3160-3163, 1998.

[24] P. D. Nellist and S. J. Pennycook, "Accurate structure determination from image reconstruction in ADF STEM," Journal of Microscopy, vol. 190, no. 1-2, pp. 159-170, 1998.

[25] E. J. Kirkland, R. F. Loane, and J. Silcox, "Simulation of annular dark field stem images using a modified multislice method," Ultramicroscopy, vol. 23, no. 1, pp. 77-96, 1987.

[26] G. Vanamu, "Study of modification of irregular titania powder passed as an aerosol through a low power microwave plasma torch and its application as a photocatalyst," M.S. thesis, University of New Mexico, Albuquerque, NM, USA, 2002.

[27] G. H. Du, Q. Chen, R. C. Che, Z. Y. Yuan, and L.-M. Peng, "Preparation and structure analysis of titanium oxide nanotubes," Applied Physics Letters, vol. 79, no. 22, pp. 3702-3704, 2001.

[28] Q. Chen, G. H. Du, S. Zhang, and L.-M. Peng, "The structure of trititanate nanotubes," Acta Crystallographica Section B: Structural Science, vol. 58, part 4, pp. 587-593, 2002.

[29] M. Anpo, "Use of visible light. Second-generation titanium oxide photocatalysts prepared by the application of an advanced metal ion-implantation method," Pure and Applied Chemistry, vol. 72, no. 9, pp. 1787-1792, 2000.

[30] S. Luo and J. L. Falconer, "Aldol condensation of acetaldehyde to form high molecular weight compounds on $\mathrm{TiO}_{2}$," Catalysis Letters, vol. 57, no. 3, pp. 89-93, 1999.

[31] J. L. Falconer and K. A. Magrini-Bair, "Photocatalytic and thermal catalytic oxidation of acetaldehyde on $\mathrm{Pt} / \mathrm{TiO}_{2}$," Journal of Catalysis, vol. 179, no. 1, pp. 171-178, 1998.

[32] W. H. Van Riemsdijk, G. H. Bolt, L. K. Koopal, and J. Blaakmeer, "Electrolyte adsorption on heterogeneous surfaces: adsorption models," Journal of Colloid and Interface Science, vol. 109, no. 1, pp. 219-228, 1986.

[33] W. H. Van Riemsdijk, J. C. M. De Wit, L. K. Koopal, and G. H. Bolt, "Metal ion adsorption on heterogeneous surfaces: adsorption models," Journal of Colloid and Interface Science, vol. 116, no. 2, pp. 511-522, 1987.

[34] L. K. Koopal and W. H. Van Riemsdijk, "Electrosorption on random and patchwise heterogeneous surfaces: electrical double-layer effects," Journal of Colloid and Interface Science, vol. 128, no. 1, pp. 188-200, 1989.

[35] C. Contescu, J. Jagiello, and J. A. Schwarz, "Chemistry of surface tungsten species on tungsten trioxide/alumina composite oxides under aqueous conditions," Journal of Physical Chemistry, vol. 97, no. 39, pp. 10152-10157, 1993. 

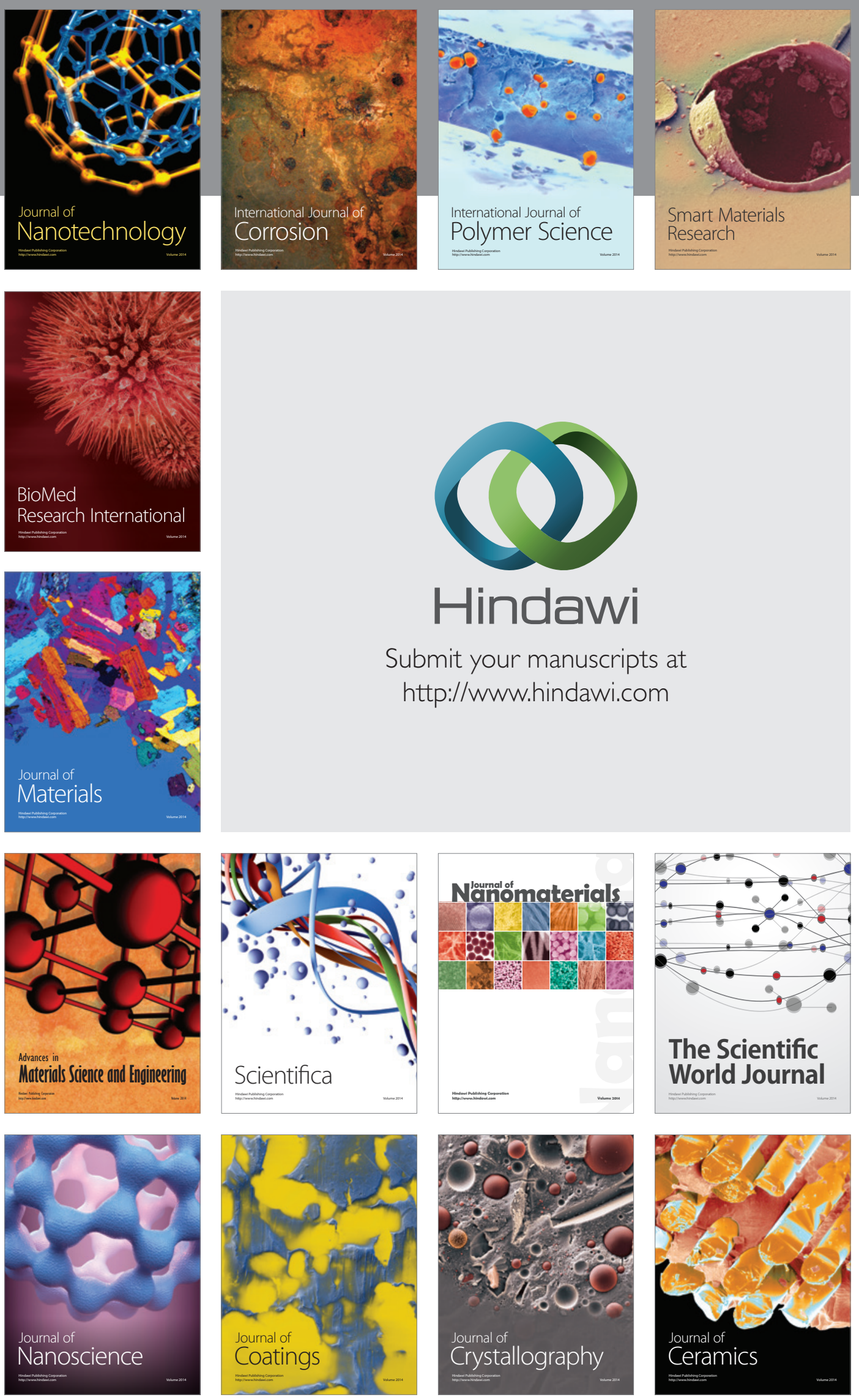

The Scientific World Journal

Submit your manuscripts at

http://www.hindawi.com

\section{World Journal}

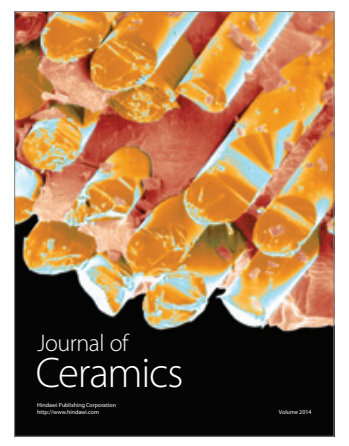

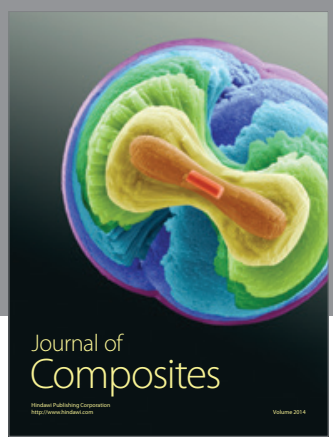
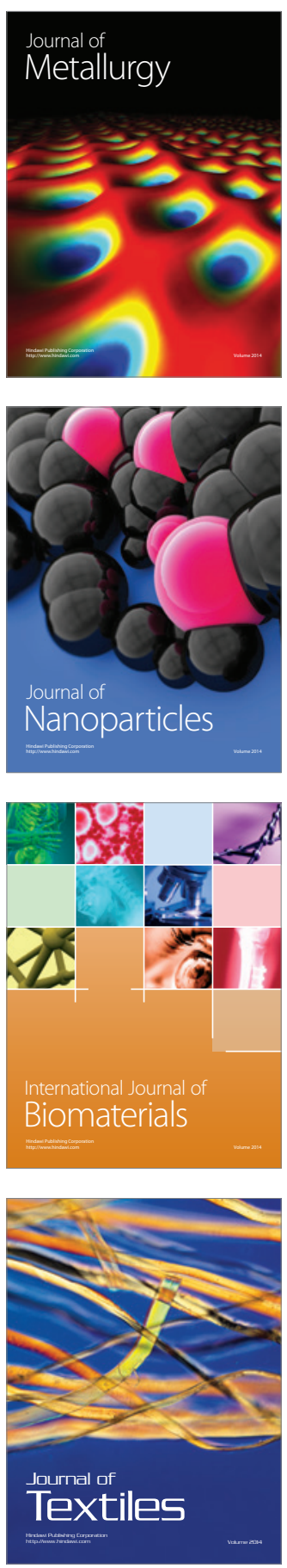